\title{
Bilateral Autologous Breast Reconstruction in a Patient with Unilateral Breast Cancer: A Case Report
}

\author{
Young Chul Kim ${ }^{1}$, Byung Ho Son ${ }^{2}$, \\ Eun Key Kim ${ }^{1}$ \\ ${ }^{1}$ Department of Plastic Surgery, Asan \\ Medical Center, University of Ulsan \\ College of Medicine, Seoul; ${ }^{2}$ Division of \\ Breast and Endocrine Surgery, \\ Department of Surgery, Asan Medical \\ Center, University of Ulsan College of \\ Medicine, Seoul, Korea
}

No potential conflict of interest relevant to this article was reported.

\begin{abstract}
Various reconstructive and/or oncoplastic options are available for breast cancer patients. In properly selected patients, autologous tissue-based reconstruction usually results in aesthetic, natural breasts. The choice of a reconstructive option for a breast cancer patient is a multifactorial decision that should consider the patient's values and preferences, as well as oncologic variables. A case of a 47-year-old woman who underwent bilateral skin-sparing mastectomy (SSM) and bilateral abdominally-based reconstruction despite having unilateral breast cancer. Right SSM and left lumpectomy were indicated for ductal carcinoma in situ in the right breast and benign tumors with microcalcifications in the left breast. The patient had very small breasts and wished for larger breasts, using her own tissue, to be created in a single-stage operation. Right SSM and left subcutaneous mastectomy were followed by bilateral free transverse rectus abdominis flap reconstruction and nipple sharing.
\end{abstract}

Keywords Breast neoplasms, Mammaplasty, Mastectomy subcutaneous

\section{INTRODUCTION}

Immediate breast reconstruction has many advantages, especially when breast skin can be preserved. The contralateral breast can either serve as a template or be modified (contralateral symmetry procedures) at the time of surgery [1]. Various reconstructive options are now available, including autologous tissue-based and prosthesis-based techniques. Autologous immediate reconstruction can usually provide esthetic, natural breasts with acceptable donor site morbidities in properly selected patients [2,3].

The indications, as well as the reconstructive techniques, should be individually and thoroughly discussed among the oncologic surgeon, the reconstructive surgeon, and the patient. We present a case in which unilateral skin-sparing mastectomy (SSM) and contralateral benign mass excision were originally planned, but the pa-

Received: Nov 14, 2017 Revised: Jan 11, 2018 Accepted: Jan 11, 2018 Correspondence: Eun Key Kim Department of Plastic Surgery, Asan Medical Center, University of Ulsan College of Medicine, 88 Olympic-ro 43-gil, Songpa-gu, Seoul 05505, Korea. E-mail: nicekek@korea.com

Copyright () 2018 The Korean Society for Aesthetic Plastic Surgery.

This is an Open Access article distributed under the terms of the Creative Commons Attribution Non-Commercial License (http://creativecommons.org/licenses/by-nc/4.0/) which permits unrestricted non-commercial use, distribution, and reproduction in any medium, provided the original work is properly cited. $\quad w w w . e-a a p s . o r g$ tient subsequently underwent bilateral SSM and abdominallybased free-flap reconstruction.

\section{CASE REPORT}

A 47-year-old Asian woman was referred to the Department of Plastic Surgery of our institution in July 2013. She had biopsy-proven ductal carcinoma in situ in the upper outer quadrant of her right breast and multiple fibroadenomas and intraductal papillomas with microcalcifications and focal typical lobular hyperplasia in the upper outer quadrant of her left breast. She did not have any family history of breast cancer. She had a height of $173 \mathrm{~cm}$, weight of 54 $\mathrm{kg}$, and a body mass index of $18.0 \mathrm{~kg} / \mathrm{m}^{2}$. Physical examination revealed very small breasts and sufficient abdominal tissue (Fig. 1).

SSM was indicated for the right breast, and simple excision of the lesion in the left breast was recommended. The patient favored autologous reconstruction and wanted to have symmetric, larger breasts if possible. She did not like the idea of a staged operation using a tissue expander. We anticipated that lumpectomy would result in major volume reduction in her left breast, which would be replaced by a prosthesis or autologous tissue. The patient preferred the idea of bilateral autologous reconstruction, understanding that the elevation of an abdominally-based free flap eliminated the possibility of a subsequent abdominally-based flap reconstruction. The 


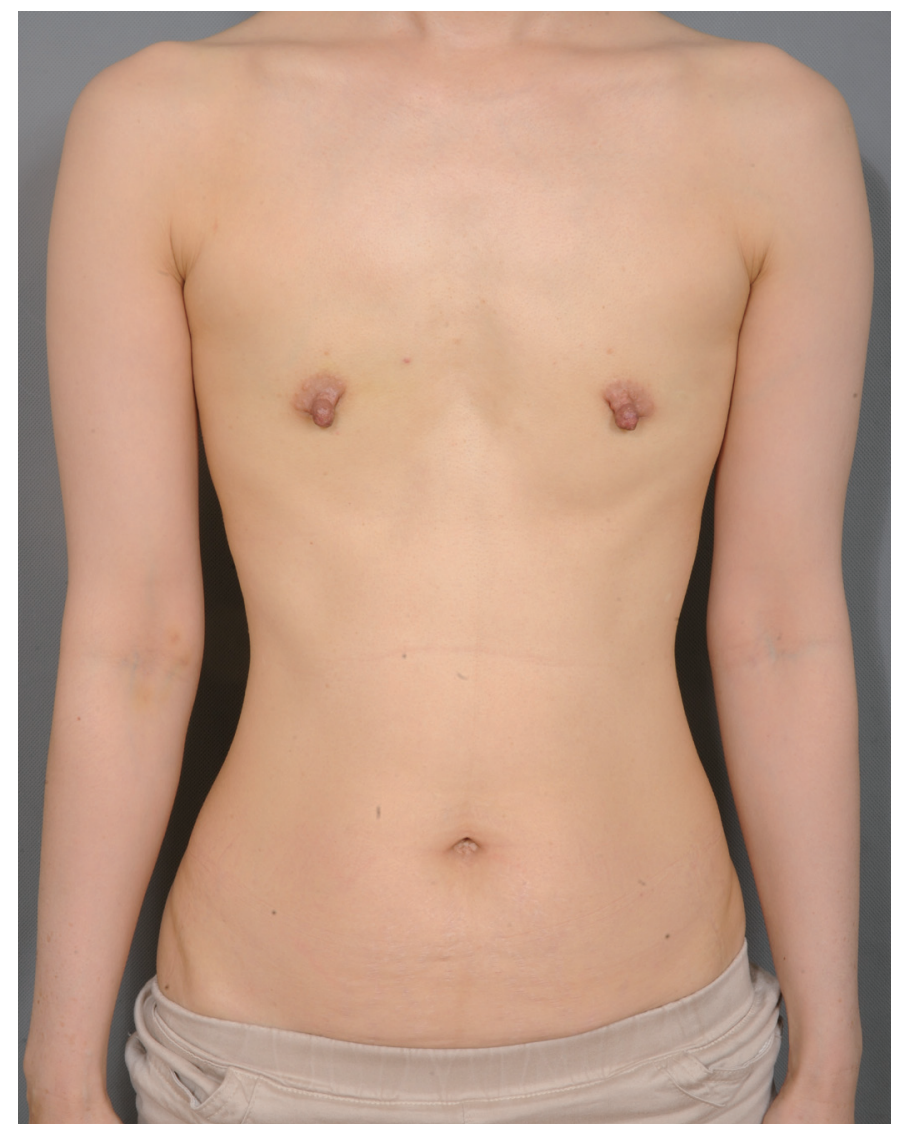

Fig. 1. Preoperative photograph of a 47-year-old patient who presented with a ductal carcinoma in situ in the right breast and multiple benign tumors with microcalcifications in the left breast.

oncologic surgeon agreed to perform contralateral mastectomy on the left breast in accordance with her wishes; bilateral total mastectomy with immediate autologous reconstruction was the final surgical plan.

At first, SSM sacrificing the nipple-areolar complex with a sentinel lymph node biopsy was performed in the right breast, followed by a contralateral nipple-areolar SSM (NASSM) in the left breast. The right and left mastectomy specimens weighed $105 \mathrm{~g}$ and $79 \mathrm{~g}$, respectively. Bilateral muscle-sparing free transverse rectus abdominis musculocutaneous (TRAM) flaps were elevated and transferred to the mastectomy defects. The right and left hemi-TRAM flaps weighed $210 \mathrm{~g}$ and $198 \mathrm{~g}$, respectively.

The right free TRAM flap was transferred to the right breast and the left flap to the left breast, with anastomoses between the deep inferior epigastric vessels and the internal mammary vessels on each side. Both flaps were inset with $180^{\circ}$ of rotation. Shape and symmetry were checked in the sitting position. Insetting and redraping of the mastectomy skin flap indicated that the use of a larger flap would have resulted in tension across the NASSM incision on the left side. The left nipple-areolar complex was excised, and the nipple was halved and simultaneously grafted onto the abdominal skin

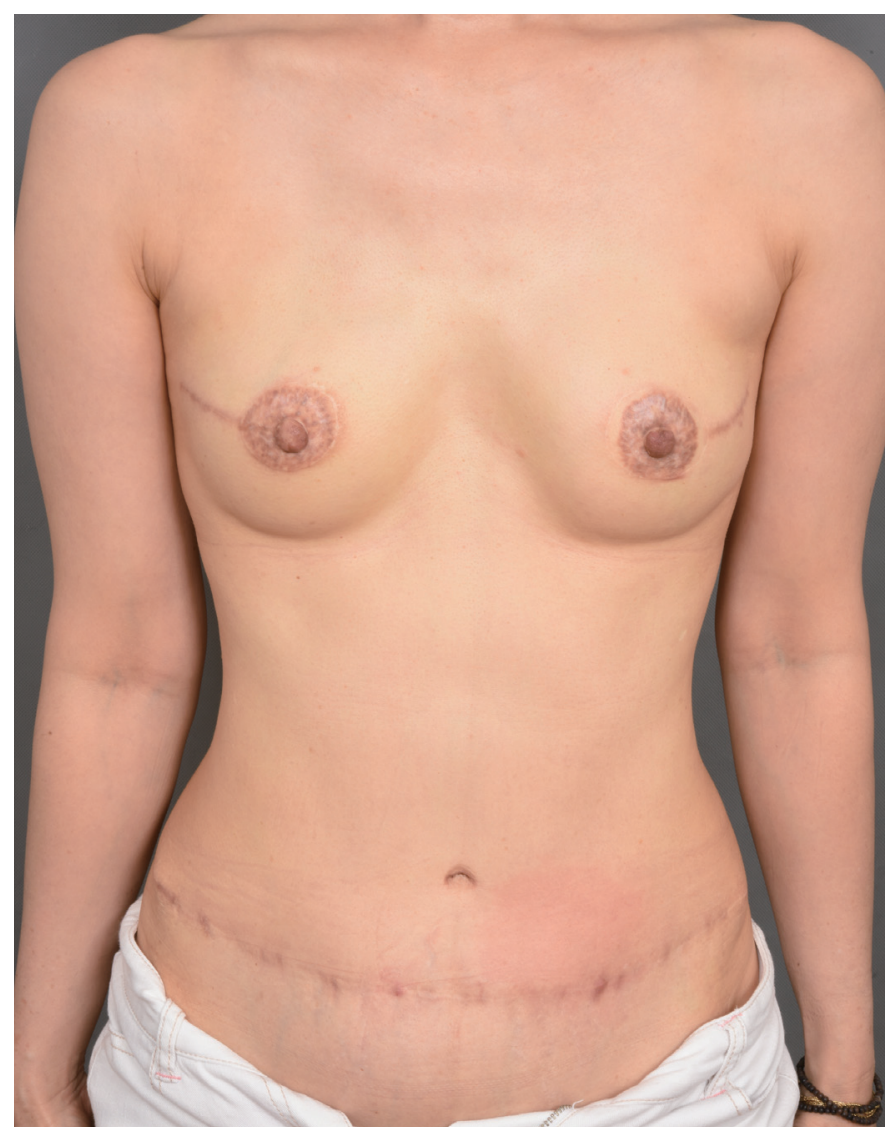

Fig. 2. Image taken 2 years post-reconstruction after bilateral skinsparing mastectomy and immediate free abdominally-based flap reconstruction with a halved nipple graft from the left side.

windows on both sides. The abdominal donor site was closed, following standard procedures. The operation time was 13 hours in total. The patient was discharged on postoperative day 7 . Tattooing of the areola was performed 4 months later in an outpatient setting.

The patient did not undergo any adjuvant therapy. After 2 years, she was generally satisfied with the results, except for some hypertrophic scarring (Fig. 2). She remained disease-free through 5 years of follow-up.

\section{DISCUSSION}

The indications of total mastectomy or breast-conserving surgery (BCS) primarily depend on the patient's oncologic status. Even for young women with breast cancer, BCS followed by whole breast radiation therapy (WBRT) is generally considered the first option whenever suitable [4]. However, BCS followed by WBRT can sometimes result in deformities of the breasts, which develop either at the time of surgery or over time [5,6]. A large lumpectomy in a relatively small breast is expected to lead to a soft tissue defect that could be managed through various oncoplastic techniques $[7,8]$. However, very small breasts preclude the use of local breast tissue 
(the volume displacement technique) in which various flaps can be utilized to add volume (the volume replacement technique) [9].

In cases of unilateral reconstruction, the contralateral breast usually serves as a template to achieve symmetry. Various contralateral procedures, such as augmentation mammoplasty, reduction mammoplasty, or mastopexy can be combined with breast reconstruction. It is not uncommon to combine contralateral augmentation with unilateral breast reconstruction in lean females who have small breasts and a slender body frame $[1,10]$. However, large-volume augmentation mammoplasty is often limited in cases of immediate reconstruction due to the tension of the mastectomy skin flap on the reconstructed side. Further, Asian skin tends to be less stretchable, and shows greater tension during wound closure [11]. In short, excessive augmentation under a limited skin envelope can result in skin necrosis and increased scarring.

Herein, we present the case of a lean Asian patient with breast cancer who required unilateral SSM and contralateral lumpectomy. She had very small involuted breasts, but sufficient abdominal tissue after 2 pregnancies and births. She desired a larger reconstruction and wanted to undergo breast reconstruction with her own abdominal tissue. She was advised that unilateral reconstruction itself would result in a larger reconstructed breast, and at first, contralateral augmentation mammoplasty using a silicone implant was discussed. However, the amount of remaining breast parenchyma after lumpectomy would be very small and the result of augmentation mammoplasty would be no better than prosthesis-based reconstruction, which the patient wanted to avoid [12]. To obtain symmetric and natural results, the patient decided to sacrifice her left (remnant) breast and undergo reconstruction with a bilateral free abdominal flap.

One-time elevation of an abdominally-based flap (a pedicled TRAM flap, a free TRAM flap, a deep inferior epigastric artery perforator flap, or even a superficial inferior epigastric artery flap) essentially eliminates any subsequent possibility of abdominally-based flap reconstruction in the future. The surgical site of any cancer recurrence after an abdominally-based flap is elevated should be reconstructed using a different flap (latissimus dorsi flap or flaps from the buttock or thigh) or prosthesis. This fact seemed to motivate the patient to undergo a bilateral mastectomy at the time of immediate breast reconstruction.

Autologous breast reconstruction has some advantages over prosthesis-based reconstruction. It usually provides softer tissue and more natural ptosis. It has also been associated with a more readily obtained final outcome and fewer secondary procedures $[1,2,13]$. Especially in small-breasted Asian women, implant-based reconstruction usually results in narrow breasts with excessive projection because small breasts tend to be wide and flat compared with most available implants [14]. Recently, we showed acceptable aesthetic results with comparable complication rates in underweight Asian women who underwent reconstruction with pedicled TRAM flaps
[14]. Autologous reconstruction can also provide a skin island when the nipple-areolar complex must be sacrificed for oncologic reasons or due to excessive tension in larger reconstructions, as in this case.

The decision to perform total mastectomy and the choice of a reconstructive technique should be made after thorough discussion among the oncologist, the oncologic surgeon, the reconstructive surgeon, and the patient. The breast cancer itself and the patient's oncologic status are the primary concerns. However, the patient's wishes should also be discussed in detail, such as autologous versus prosthesis-based reconstruction, a one-stage operation versus a staged operation, and whether or not to perform a contralateral procedure, among other factors. Most bilateral mastectomies with contralateral prophylactic mastectomy are performed in patients who are at risk of developing contralateral cancer; although the decision in this case was made mainly for esthetic reasons, rather than oncologic needs.

A contralateral balancing procedure, especially augmentation mammoplasty, can usually be performed using a prosthesis. However, in selected patients, as in this case, autologous total reconstruction can result in better aesthetic results, especially when WBRT following BCS would otherwise be indicated.

In conclusion, bilateral total autologous reconstruction can be considered as a viable option when unilateral mastectomy and contralateral lumpectomy are indicated in selected patients with very small breasts who desire a larger reconstruction with autologous tissue in a single-stage operation.

\section{PATIENT CONSENT}

Patient provided written consent for the use of her images.

\section{REFERENCES}

1. Duggal CS, Grudziak J, Metcalfe DB, et al. The effects of breast size in unilateral postmastectomy breast reconstruction. Ann Plast Surg 2013; 70:506-12.

2. Leone MS, Priano V, Franchelli S, et al. Factors affecting symmetrization of the contralateral breast: a 7-year unilateral postmastectomy breast reconstruction experience. Aesthetic Plast Surg 2011;35:446-51.

3. Kim EK, Suh YC, Maldonado AA, et al. Patients' aesthetic concerns after horizontally placed abdominal free flap breast reconstruction. Aesthetic Plast Surg 2015;39:686-93.

4. Vila J, Gandini S, Gentilini O. Overall survival according to type of surgery in young $(</=40$ years) early breast cancer patients: a systematic meta-analysis comparing breast-conserving surgery versus mastectomy. Breast 2015;24:175-81.

5. Bajaj AK, Kon PS, Oberg KC, et al. Aesthetic outcomes in patients undergoing breast conservation therapy for the treatment of localized breast cancer. Plast Reconstr Surg 2004;114:1442-9. 
6. Losken A, Pinell-White X, Hodges M, et al. Evaluating outcomes after correction of the breast conservation therapy deformity. Ann Plast Surg 2015;74 Suppl 4:S209-13.

7. Munhoz AM, Montag E, Filassi JR, et al. Current approaches to managing partial breast defects: the role of conservative breast surgery reconstruction. Anticancer Res 2014;34:1099-114.

8. Munhoz AM, Montag E, Gemperli R. Oncoplastic breast surgery: indications, techniques and perspectives. Gland Surg 2013;2:143-57.

9. Lee JW, Kim MC, Park HY, et al. Oncoplastic volume replacement techniques according to the excised volume and tumor location in smallto moderate-sized breasts. Gland Surg 2014;3:14-21.

10. Losken A, Carlson GW, Bostwick J 3rd, et al. Trends in unilateral breast reconstruction and management of the contralateral breast: the Emo- ry experience. Plast Reconstr Surg 2002;110:89-97.

11. Tan BK, Chim H, Ng ZY, et al. Aesthetic design of skin-sparing mastectomy incisions for immediate autologous tissue breast reconstruction in asian women. Arch Plast Surg 2014;41:366-73.

12. Stokes RB. Breast augmentation in thin women: patient satisfaction with saline-filled implants. Aesthetic Plast Surg 2004;28:153-7.

13. Eltahir Y, Werners LL, Dreise MM, et al. Which breast is the best? Successful autologous or alloplastic breast reconstruction: patient-reported quality-of-life outcomes. Plast Reconstr Surg 2015;135:43-50.

14. Kim EK, Eom JS, Hwang $\mathrm{CH}$, et al. Immediate transverse rectus abdominis musculocutaneous (TRAM) flap breast reconstruction in underweight Asian patients. Breast Cancer 2014;21:693-7. 